\title{
INTERVENCIÓNY MEDIACIÓN SOCIAL. DEFINICIÓN Y CONTEXTOS PROFESIONALES
}

\section{INTERVENTION AND SOCIAL MEDIATION. DEFINITION AND PROFESSIONAL CONTEXT}

\author{
Concepción Castro Clemente \\ Profesora Tutora de Trabajo Social. Universidad Nacional de \\ Educación a Distancia
}

\begin{abstract}
Resumen: El funcionamiento de la sociedad está directamente interrelacionado con el bienestar de sus ciudadanos, cualquier cambio o transformación social repercute, directa o indirectamente, en la población. Son muchos los elementos que interactúan en el sistema social y que no siempre tienen efectos positivos en el conjunto social cuando se produce un cambio. Emergen, por tanto, nuevos escenarios de conflicto o de necesidad en el sistema social que demandan estrategias y técnicas de protección para enfrentar y conseguir el equilibrio y bienestar de las personas, grupos y comunidades de forma precisa y eficaz. El objetivo de la intervención social es rehabilitar y mediar en las situaciones problemáticas para encarar el complejo fenómeno de la exclusión. Mediante un proceso metodológico y la utilización de técnicas de mediación profesional, se ponen en marcha un conjunto de acciones para cubrir las necesidades humanas que permitan a las personas desarrollarse integralmente y mejorar su calidad de vida.
\end{abstract}

Palabras clave: Intervención Social, Mediación Social, Exclusión Social, Metodología de Intervención, Mediador Social.

Abstract: The functioning of society is directly interrelated with the welfare of its citizens, any change or social transformation has a direct or indirect impact on the citizenship. There are many elements that interact in the social system and that do not always have positive effects on the social set when a change occurs. Therefore, emerge newscenarios of conflict or of necessity in the social system that demand strategies and techniques of protection to deal and get the balance and welfare of individuals, groups and communities accurately and effectively. The objective of social intervention is to rehabilitate and mediate in problematic situations to deal the complex phenomenon of exclusion. Through a methodological process and the use of 
professional mediation techniques a set of actions are implemented to meet the human needs that allow people to develop fully and improve their quality of life.

Key words: Social Intervention, Social Mediation, Social Exclusion, Intervention Methodology, Social Mediator.

\section{Introducción}

En las últimas décadas, la sociedad ha experimentado grandes transformaciones sociales. La crisis económica, los cambios de las estructuras sociales, los retos de la globalización capitalista, la sucesión de reformas políticas así como las modificaciones experimentadas por la familia en la que emergen nuevos entornos y modelos de convivencia familiares más libres y plurales, presentan nuevas necesidades en el conjunto social.

Estos nuevos escenarios demandan una respuesta social para enfrentar y conseguir el equilibrio y bienestar de las personas, grupos y comunidades de forma precisa y eficaz. Sin duda, hay que intervenir y elaborar estrategias y técnicas específicas para conseguir un modelo de convivencia socialmente óptimo.

El concepto de intervención social se define como "toda actividad profesional consciente, organizada y planificada y dirigida a actuar sobre una realidad social para estudiarla, analizarla, modificarla y cambiarla en la consecución de una mejora positiva" (Fernández, De Lorenzo y Vázquez, 2012). Toda acción de intervención, se asienta en una doble alianza entre en una base teórica, que la fundamente, así como en un método científico que permita cambiar la situación social.

A partir de esta visión globalizadora en la que le intervención social se basa en un proceder científico-metodológico, es fundamental la aplicación de técnicas específicas para la resolución de cada problema en su contexto particular. La mediación social sería una de las técnicas especializadas en la gestión y resolución de conflictos sociales, familiares, penales, interculturales, judiciales, educativos o comunitarios.

El concepto de mediación es definido por el Diccionario de Trabajo Social (2012) como el "proceso de resolución negociada y consensuada entre partes enfrentadas bajo la actuación de un mediador, promotor de una solución satisfac- 
toria y constructiva”. Esta técnica aplicada en el ámbito de intervención social, presenta las siguientes características según Martín (2012):

- Es un tratamiento de los conflictos más completo y eficaz desde una visión interdisciplinar.

- Tiene un objetivo concreto y preciso.

- Su carácter es flexible y ausente de formalismos.

- Favorece las soluciones y voluntario cumplimiento de las partes afectadas.

- Propicia soluciones adaptadas a las necesidades de las personas, es decir, para las partes implicadas.

- Los acuerdos alcanzados en la mediación presentan mayor durabilidad.

- Es una herramienta especialmente eficaz en aquellos conflictos en los que sus protagonistas habrán de seguir manteniendo relación entre sí.

El empleo de esta técnica es desarrollada por profesionales debidamente cualificados conforme a los principios, procedimientos y deontología de la mediación. Las acciones del mediador están caracterizadas por su imparcialidad y neutralidad, facilita la comunicación, acuerdo y negociación en la resolución de situaciones problemáticas. Asimismo, representa una especialización en el cuadro de profesiones de intervención en conflictos sociales en la vasta y multifactorial conflictividad de nuestro tiempo.

Son muchas las situaciones que pueden requerir una intervención social, abordarlos implica ubicarlo dentro del contexto en el que el individuo, o comunidad, expresa su malestar. Así, se podrían encontrar diferentes intervenciones centradas en distintas necesidades sociales sobre la exclusión, el género, la educación, la marginación, la pobreza, el impacto social, etc.

Si bien son muchas las circunstancias que requieren de una intervención y mediación profesional, el ámbito familiar, escolar y comunitario así como la exclusión social representan los principales contextos de intervención. 
A continuación se analizarán los principales contextos profesionales donde la mediación está siendo un elemento imprescindible para los profesionales vinculados con la intervención social.

\section{Ámbito familiar}

El espacio principal en el desarrollo psicológico y de apoyo para los menores es la familia, en ella se aprende la mayor parte de las conductas sociales y emocionales. Es una red de comunicación entrelazada donde todos los miembros se influyen entre sí. Desde la cuna los niños están sometidos a ese proceso.

El grupo familiar dispone de una estructura que atiende a la interacción interna de sus integrantes, pero también a la interacción externa con el medio que les rodea. En las últimas décadas, la familia ha experimentado grandes cambios. La irrupción de repetidas crisis económicas ha impactado en el grupo familiar por el que se ven obligados a trabajar todos sus miembros para mantener la economía familiar.

Este cúmulo de circunstancias son origen de muchas fuentes de conflicto que afectan a las dinámicas familiares: rupturas conyugales y divorcios; dificultades laborales e inestabilidad económica; larga dependencia material de los hijos del grupo familiar; malestar psicológico de los padres por su situación y dificultad en las propuestas de ocio en un mercado de consumo y en el que prevalece el uso de las nuevas tecnologías (Fernández-Crehuet, 2016).

Adicionalmente a estas situaciones, se le añade la dificultad de conciliar el trabajo y la familia y que está derivando en padres sobrecargados y estresados en el que la interacción intrafamiliar aparece más frágil, así como la falta de tiempo para atender, dedicar o escuchar a sus hijos.

La intervención familiar, caracterizada por la diversidad de estrategias y modalidades de intervención en el que un mismo problema se afronta de manera distinta en familias diferentes, requiere sea analizada de manera individualizada y desde la flexibilidad a fin de adaptarse a las distintas situaciones y particularidades que puedan presentar (Fernández y Ponce de León, 2011; Moreno, 2014). 
Tomando como referente el modelo elaborado por los autores Fernández y Ponce de León (2011), se presenta el procedimiento metodológico de intervención con familias estructurado en seis etapas: 1. Acogida de la problemática familiar y recogida de la información, 2. Diagnóstico social, 3. Diseño de intervención, 4. Elaboración de un plan de acción, 5. Aplicación del diseño o plan de acción, y 6. Evaluación de los resultados conseguidos. Es a partir de la fase de diseño cuando los profesionales valoran el incluir o no la mediación como técnicas para resolver el conflicto planteado por la familia, pues no en todos los casos es viable su aplicación.

El proceso de intervención familiar se inicia con la primera toma de contacto con la familia y en el que se explicita el problema o conflicto en el sistema familiar. Posteriormente, y siguiendo el protocolo de actuación, se recopila de manera cordial y receptiva información de todos los miembros para poder elaborar el diagnóstico.

Una vez identificadas las dificultades y necesidades de la familia, se procede de manera reflexiva, analítica y racional a la síntesis y valoración de los datos. No sólo se analiza la estructura que mantiene el problema, sino también los factores de riesgo y de protección así como los recursos que dispone la familia para afrontar la situación y propiciar el cambio. Esta aproximación con la familia permite elaborar las hipótesis de trabajo y determinar los cambios necesarios para transformar la problemática que experimenta.

Cada familia es única, por esta razón, el diseño de intervención debe estar adaptado en función a sus necesidades implícitas y explícitas. En el plan de acción se definen las metas y objetivos que se pretenden alcanzar marcando, para ello, el plazo de consecución previsto para conseguirlos en días, semanas y meses. En esta fase, además de que la familia puede visualizar una proyección de futuro reduciendo así su incertidumbre, también posibilita evaluar los progresos y cambios logrados. Es en esta etapa donde el profesional podrá decidir si utilizar la mediación, bien como una técnica más de intervención social, o bien como procedimiento extrajudicial en el caso de separaciones, divorcios, tutelas, etc. Esta distinción es importante pues la mediación en el primer caso podría permitir, por ejemplo, llegar a un acuerdo en los horarios de salidas de los hijos. En cuanto a los procesos conflictivos en las parejas, y guiados por un profesional neutral, imparcial y cualificado, podrían negociar y decidir, por sí mismas, todos los efectos de su ruptura. 
El cierre del proceso de intervención se realiza con la evaluación. Es el momento en el que se analizan los resultados y se valora la eficacia y efectividad de la intervención con la familia. Aparte de revisar los cambios observados, se evalúa el proceso en sí mismo y el grado de consecución de los objetivos determinados en el diseño de intervención. Para ello, es recomendable se utilicen técnicas cualitativas y cuantitativas con el fin baremar con mayor precisión los resultados.

\section{Contexto escolar}

La escuela es un espacio social y de relación en el que, además de los contenidos académicos, se proporciona al alumnado una experiencia de relación que tiene gran trascendencia en los demás espacios sociales.

La convivencia escolar es el gran reto, es el escenario social en el que se dan continuas interacciones y en el que el estudiante permanece gran parte de su tiempo pero también es uno de los ámbitos donde se originan conflictos y se desarrollan conductas problemáticas.

Existen una serie de factores como son, entre otros, el fracaso escolar, la actitud negativa ante el trabajo, un desmedido estrés, la impotencia e indefensión que pueden desencadenar en comportamientos de riesgo en los menores.

Estas conductas se pueden definir como "aquellas comportamientos que resultan atractivos por el riesgo y por las sensaciones que produce enfrentarse con el peligro" (Castro y Rodríguez, 2016) y entre las que se encuentran la violencia (acoso y ciberacoso), el consumo de sustancias adictivas (alcohol, tabaco y cannabis), delincuencia o trastornos de alimentación.

El problema del consumo de sustancias adictivas también está presente en el proceso educativo de manera directa (borracheras en una fiesta) o indirecta (incidentes en el barrio, inadaptación escolar de un alumno), en los hogares de los estudiantes, entornos cercarnos y medios de comunicación social. Aunque existen campañas de información y persuasión sobre los efectos nocivos de su consumo, es la comunidad educativa la que presenta el mejor recurso en prevención y reducción del uso de drogas. 
En cuanto a las manifestaciones de violencia en el ámbito educativo, las consecuencias, tanto para estudiantes como para docentes, son de impotencia, inseguridad, angustia, intranquilidad y desesperanza. Desestabilizan a la comunidad educativa y debilitan las formas instituidas de solidaridad y confianza.

La escuela representa uno de los mejores recursos para intervenir en los comportamientos violentos o disruptivos de los estudiantes así como en su aptitud frente a las drogas. A través de la información, estrategias de intervención y actividades escolares brinda la oportunidad de mejorar los estilos de afrontamiento a fin de prevenir posibles alteraciones de tipo emocional ligadas a los cambios sociales, físicos y psicológicos del alumnado (Cagigal de Gregorio, 2009; De Bofarull, 2013; Castro, 2016; Estévez y Musitu, 2016).

La labor de los tutores es crucial para prevenir e intervenir sobre los comportamientos desajustados y de riesgo de los estudiantes. Además de la labor principal de impartir formación humana, son los que mejor conocen la organización del grupo, relaciones de poder, afectividad, valores, rendimiento escolar y problemas específicos de los alumnos.

La intervención escolar está íntimamente relacionada con la capacidad de atención que los padres dediquen a sus hijos. La implicación y tiempo que destinen en el bienestar y desarrollo del menor tiene una repercusión importante en la probabilidad de desarrollar conductas problemáticas, comportamientos de riesgo y rendimiento formativo del menor.

Afrontar situaciones de riesgo y de conflicto escolar requiere un trabajo en equipo de todos los colectivos que integran la comunidad educativa para disponer de estrategias, protocolos de intervención, planes de prevención y sistemas de mediación con la finalidad de resolver el conflicto, prevenir conductas de riesgo y generar pautas de buena convivencia (Castro, 2016).

Si estas situaciones no son detectadas en sus fases iniciales, ni a tiempo que permitan poner en marcha una intervención preventiva o reparadora adecuada, pueden agravarse y ocasionar situaciones de desprotección infantil y juvenil grave, importantes daños emocionales así como afectar a su desarrollo psíquico-físico. 


\section{Conflicto comunitario}

El concepto de comunidad puede definirse "como un conjunto de personas que viven juntas bajo ciertas reglas o que tienen los mismos intereses: idioma, costumbres, valores, tareas, visión del mundo, edad, ubicación geográfica, estatus social o roles” (Fernández y Ponce de León, 2014).

La intervención comunitaria se pone en funcionamiento cuando se detecta un problema social que les afecta colectivamente. A partir de ese momento, se emprenden un conjunto de acciones que tienen como objetivo promover la participación activa de los actores sociales a fin de lograr un cambio estructural para la mejora de la calidad de vida de todos los ciudadanos.

A través de la acción social, la reflexión, la movilización y la capacidad de decisión, la comunidad adquiere el protagonismo en abordar y transformar las situaciones de desigualdad que les afecta.

Los puntos fundamentales que deben abordarse en la intervención social comunitaria, podemos tomar como referencia el trabajo elaborado por Fernández y Ponce de León (2014) en el que determinan cinco fases cronológicas relacionadas entre sí: 1. Análisis de la situación de la comunidad y delimitación del problemas con repercusiones colectivas; 2 . Autodiagnóstico de la comunidad, 3. Elaboración de propuestas y retos: proyecto racional de cambio; 4. La comunidad puesta en marcha: avances, retrocesos, conflictos, poder y negociación de la comunidad, y 5 . Evaluación continuada y final: logro de metas y aumento de la densidad relacional de la comunidad.

Desde el punto de vista histórico, la intervención en la comunidad estaba ligada a los Servicios Sociales, sobre todo tenían carácter asistencial. En la actualidad, son diversas las disciplinas que comparten el interés en mejorar el bienestar y la calidad de vida comunitaria como Trabajo Social, Psicología, Derecho o Pedagogía, entre otras (Fundación Foeesa, 2009; González, 2014).

En cuanto a los ámbitos de intervención que se abordan, y lejos de ser un servicio meramente asistencial, pueden relacionarse diferentes campos de trabajo como: familias destructuradas, exclusión social, inmigración, personas mayores o violencia machista (Estévez y Musitu, 2016). 
En nuestro país, la intervención comunitaria se desarrollan en instituciones públicas, entidades privadas y diferentes Organizaciones No Gubernamentales, además de los Centros de Servicios Sociales. Esta heterogeneidad de ámbitos de competencias favorece la existencia de muchos y muy diferentes programas de intervención y de prevención.

\section{La exclusión social}

En las últimas décadas se ha vivido un crecimiento económico sin precedentes, pero esta situación también ha dejado al margen a un elevado número de personas que se ven imposibilitadas a acceder a los mismos servicios que el resto. Esta realidad, y tras las reiteradas crisis económicas, ha incrementado, todavía más, el riesgo de exclusión social, las necesidades sociales han aumentado pero los recursos para satisfacer estas demandas han disminuido, actualmente son escasos y limitados.

En este proceso de alejamiento progresivo de una situación de integración social, pueden distinguirse una acumulación de barreras en distintos ámbitos: laboral, formativo, sociosanitario, económico, relacional, habitacional y de acceso limitado a los mecanismos de protección (Fundación FOESSA, 2009).

Los programas de formación y el acceso al empleo son considerados como dos valiosos recursos de lucha contra la exclusión desde el ámbito de la intervención social. Por ello, se han desarrollado una elevada heterogeneidad de estrategias de activación de empleo dirigidas a la población más excluida.

El acceso al mercado laboral representa la fase más significativa de intervención en la inclusión social, constituye uno de los primeros pasos del proceso de integración pero en el que también se persiguen otros objetivos. Además de garantizar unos ingresos para dar cobertura a las necesidades más básicas de las personas en situación de dificultad, constituye un instrumento en el que también puede abordarse otros situaciones personales como es el aumento de la autoestima, la adquisición de habilidades o la mejora de las relaciones sociales.

Sin embargo, en la actualidad, en un contexto de crisis económica en el que los programas de activación adquieren protagonismo pero en el que la esca- 
sez de empleo es patente, el tiempo de espera para acceder al mercado laboral así como la derivación a recursos de formación y orientación, inciden de manera negativa en las personas que viven esta situación. Algunos adquieren actitutes de fracaso, sienten que no están lo suficientemente preparadas ni capacitados para el puesto y que su situación no va a cambiar.

En los últimos años, han surgido numerosas iniciativas de empleo impulsadas desde el sector no lucrativo: asociaciones, empresas sociales, centros ocupacionales, proyectos de inserción... Bajo estas fórmulas de oportunidades laborales, no sólo se pretende incrementar el acceso al mercado laboral sino que se trata de conciliar las oportunidades de empleo considerando las especiales características de los participantes en el diseño de estrategias de activación y mediación.

Desde la intervención social, en el que cada vez más se tiene el convencimiento que la situación de las persona en exclusión social no sólo tiene su origen en la falta de ingresos económicos sino que también incluye otras situaciones problemáticas como la formación, la vivienda, la salud (física y mental), las relaciones sociales, la autovalía personal, el apoyo social, la composición del hogar o la pertenencia a minorías, entre otros factores, el diseño de intervención adquiere un carácter multidimensional dirigidas a superar estas situaciones y conflictos, y en donde la mediación ocupa un lugar destacado.

Es por ello, y a fín de mejorar la eficacia de cualquier programa de activación de empleo propuestos desde la administración pública o sector privado, toda acción destinada a la superación de estas situaciones de exclusión debe incluir, además de la garantía de ingresos y el acceso al empleo, intervenciones de acompañamiento y apoyo social destinadas dirigidas a la superación del resto de dificultades y conflictos personales y familiares.

\section{Conclusiones}

Es evidente que vivimos en un mundo de continuas e imparables transformaciones sociales. Los cambios que se han producido en las últimas décadas -globalización, crisis económica, desempleo, acceso de la mujer al mercado laboral, horarios extensos de la jornada laboral o transformaciones familiares- han 
generado diferentes problemáticas y conflictos en nuestra vida cotidiana que requieren de intervención y mediación social.

Son muchas las circunstancias, contextuales y circunstanciales, que pueden poner al individuo, o a la comunidad, en situaciones de vulnerabilidad, de exclusión o en riesgo social que requieren de una intervención profesional o de la mediación de un tercero para sobrellevar un acontecimiento estresante. No hay que resolver una cuestión, sino varias que precisan poner en marcha una serie de estrategias específicas para solucionarlo, hay que intervenir.

El contexto de la intervención en lo social debe ubicarse en el escenario en el que se está produciendo esos choques y enfrentamientos que son generadores de una multiplicidad de conflictos. El objetivo de la intervención es la de modificar, interceder y formar parte en un problema de riesgo social, particular o comunitario, a fin de cubrir las necesidades humanas que permitan a las personas desarrollarse integralmente y mejorar su calidad de vida.

Los motivos por los que se demanda una intervención social son muchos y diferentes. Algunos pueden ser para la descarga emocional de la persona, otros para atender una necesidad implícita de la colectividad en un determinado contexto social, como también para la elaboración e implementación de medidas específicas en la resolución de una situación conflictiva en un ámbito concreto. Necesidades y problemas que deben resolverse con el apoyo de las instituciones públicas y privadas a través de los recursos económicos, sociales, educativos o políticos.

La intervención social participa en todos los sectores de la sociedad, implanta recursos y aplica una metodología en el que se abordan diferentes dimensiones: personas, grupos o comunidad. Mediante el desarrollo de un proceso de estrategias, programas y técnicas orientadas a la prevención y protección, se pretende modificar procesos de exclusión social así como promover acciones que favorezcan la inclusión, la inserción e integración social de todas las personas.

En cuanto a las técnicas especializadas que emplean los profesionales de intervención, la mediación constituye su principal herramienta de trabajo para favorecer la resolución de los conflictos sociales. A través de ella, y dentro de un contexto natural y cercano en el que se establece una relación participativa entre 
los distintos sujetos, se establece una relación de apoyo profesional en la búsqueda de soluciones a los distintos problemas planteados.

Esta técnica representa un incuestionable recurso en distintos ámbitos para el tratamiento y resolución de conflictos: social, jurídico, familiar, educativo, comunitario o penal, entre otros. Su carácter flexible y con unos objetivos precisos y concretos, favorece el acuerdo de soluciones entre las personas implicadas.

\section{Bibliografía}

CAGIGAL DE GREGORIO, V. (2009). La orientación familiar en el ámbito escolar. Madrid: Universidad Pontificia Comillas y Obra Social Caja Madrid.

CASTRO, C. (2016). El fenómeno de la violencia entre iguales en España. Roles, género, edad, actitudes y estrategias de intervención. Revista Catedra Paralela, 13, 127-154.

CASTRO, C., y RODRÍGUEZ, E. (2016). Intervención social con adolescentes: Necesidades y recursos. Revista Trabajo Social Hoy, 77, 7-23. DOI 10.12960/TSH.2016.0001.

DE BOFARULL, I. (2013). Fortalezas y competencias de la familia. Bases para la orientación y mediación familiar. Barcelona: Instituto de Estudios Superiores de la Familia (IESF). Universitat Internacional de Catalunya.

ESTÉVEZ, E., y MUSITU, G. (Coords.). (2016). Intervención psicoeducativa en el ámbito familiar, social y comunitario. Madrid: Ediciones Paraninfo, S.A.

FERNÁNDEZ, T., y PONCE DE LEÓN, L. (2011). Trabajo Social con familias. Madrid: Ediciones Académicas, S.A.

FERNÁNDEZ, T., y PONCE DE LEÓN, L (2012). Trabajo Social individualizado. Madrid: Ediciones Académicas S.A.

FERNÁNDEZ, T., y PONCE DE LEÓN, L (2014). Nociones básicas de Trabajo Social. Madrid: Ediciones Académicas, S.A.

FERNÁNDEZ, T., DE LORENZO, R., y VÁZQUEZ, O. (2012). Diccionario de Trabajo Social. Madrid: Alianza Editorial, S.A,.

FERNÁNDEZ-CREHUET, J. (2016). La conciliación de la vida profesional, familiar y personal. España en el contexto europeo. Madrid: Ediciones Pirámide (Grupo Anaya, S.A.).

FUNDACIÓN FOESSA. (2009). Actuar ante la exclusión. Análisis, políticas y herramientas para la inclusión social. Madrid: Fundación FOESSA y Cáritas Española Editores.

GONZÁlEZ, A. (2014). Sujetos en la intervención social. Madrid: Cáritas Española Editores.

MARTÍN, A. (2012). Mediación en conflictos versus mediación en Trabajo Social. Revista Trabajo Social Hoy, 65, 7-14.

MORENO, A. (2014). Manual de Terapia Sistémica. Principios y herramientas de intervención. Bilbao: Desclée de Brouwer. 